\title{
INFN
}

Istituto Nazionale di Fisica Nucleare

\section{Gaussino - a Gaudi-based core simulation framework}

\section{Benedetto G. Siddi(1), D. Müller(2)}

On behalf of the LHCb experiment

(1)INFN - Sezione Ferrara

(2)CERN

\section{IEEE/NSS 2019}

Manchester, UK

B. Siddi

INFN Ferrara 


\section{Motivation}

- Starting point: general modernisation of LHCb software

- Main concept: separate core functionality for simulation

- Experiment-independent framework: LHCb based algorithm built on top of that

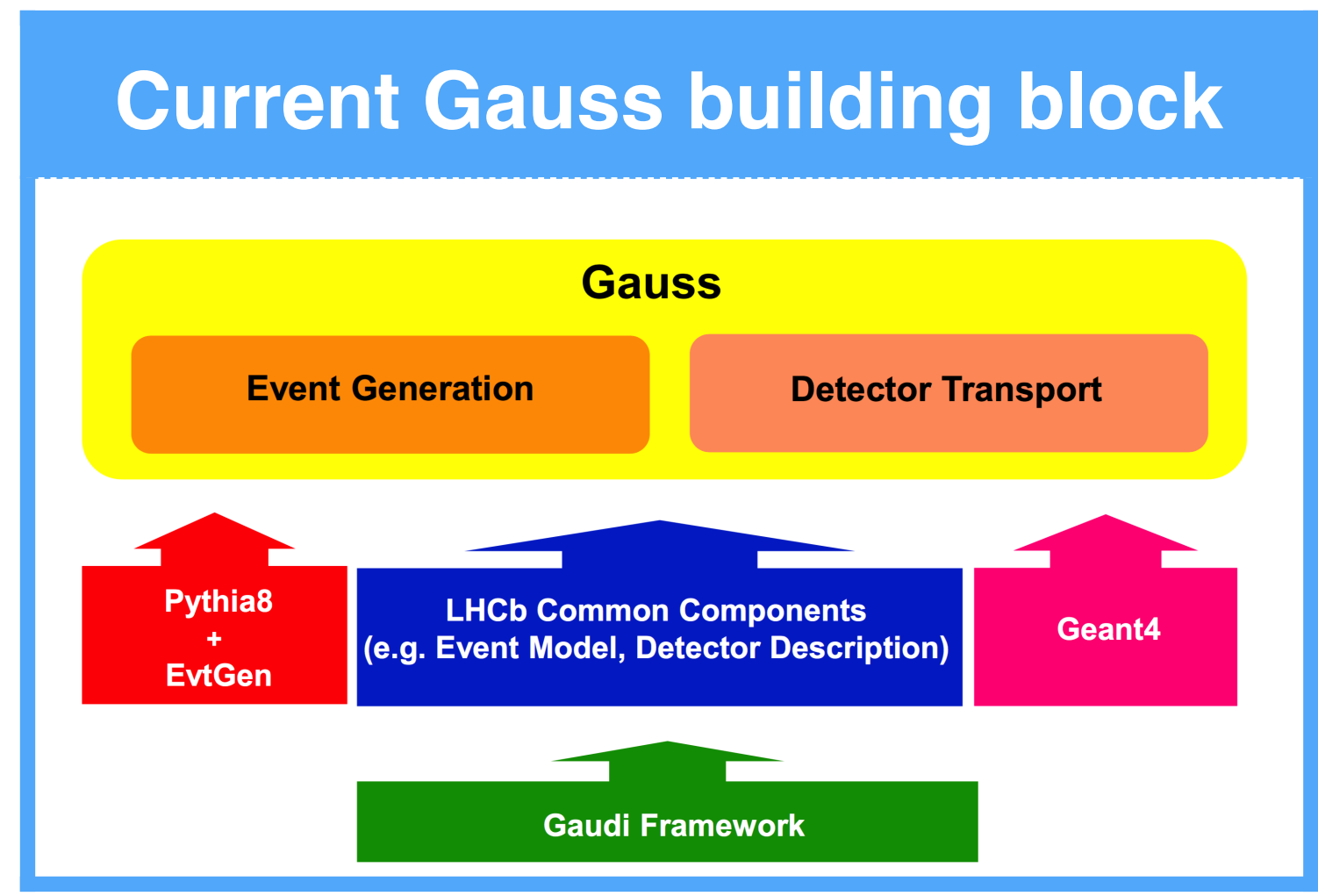

- 15 years old

- Single-threaded framework

- Memory usage becoming an issue

- Simulation based on Geant4

- Increase in CPU doesn't translate into an increase in memory 


\section{Main Features}

- Keep the design choices of current Gauss, e.g.:

- Integrated generation and simulation phase

- Include the following features:

- Multi-threaded Gaudi

- Can benefit from MT generators

- HepMC3

- Improve easy python configuration for jobs

- Multi-threaded Geant4

\section{New Framework built dependencies}

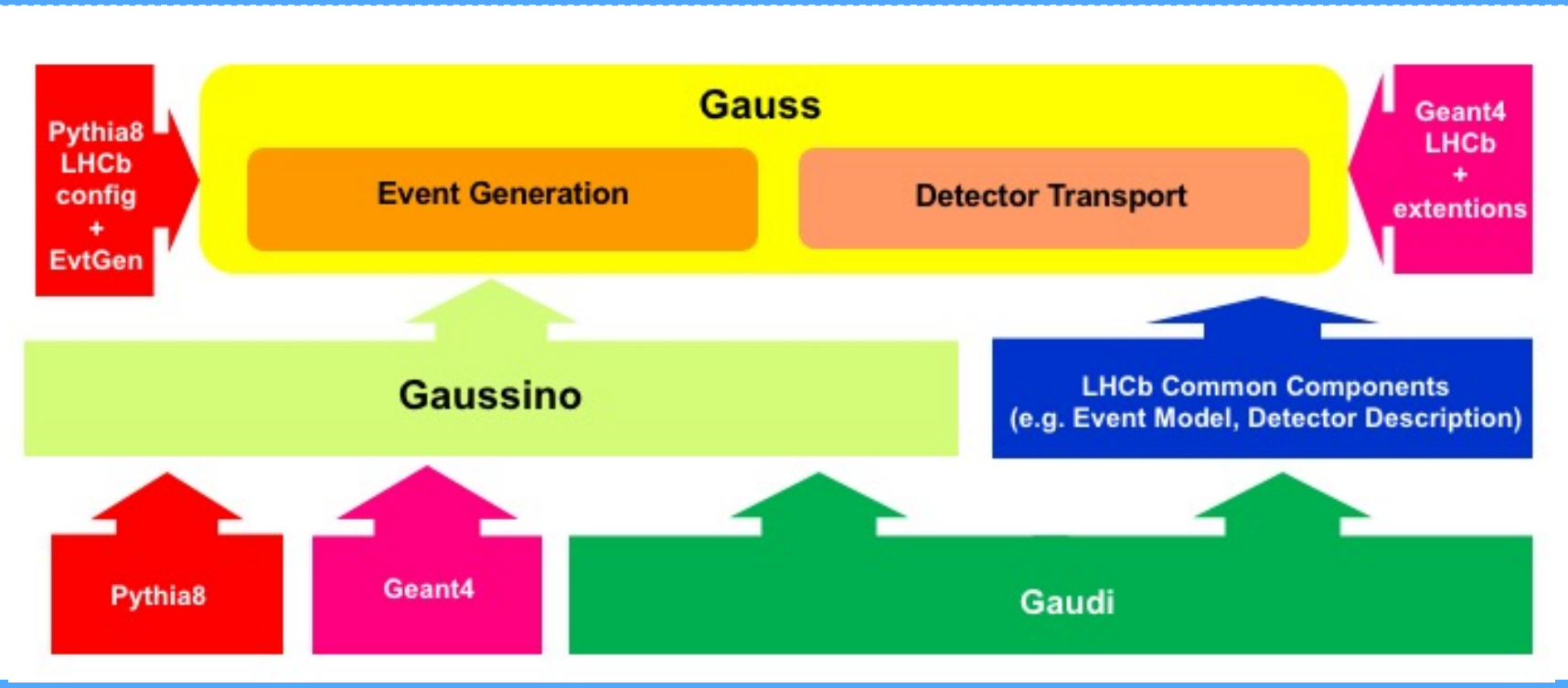




\section{Gaussino}

- A complete simulation framework following the basic Gauss architecture

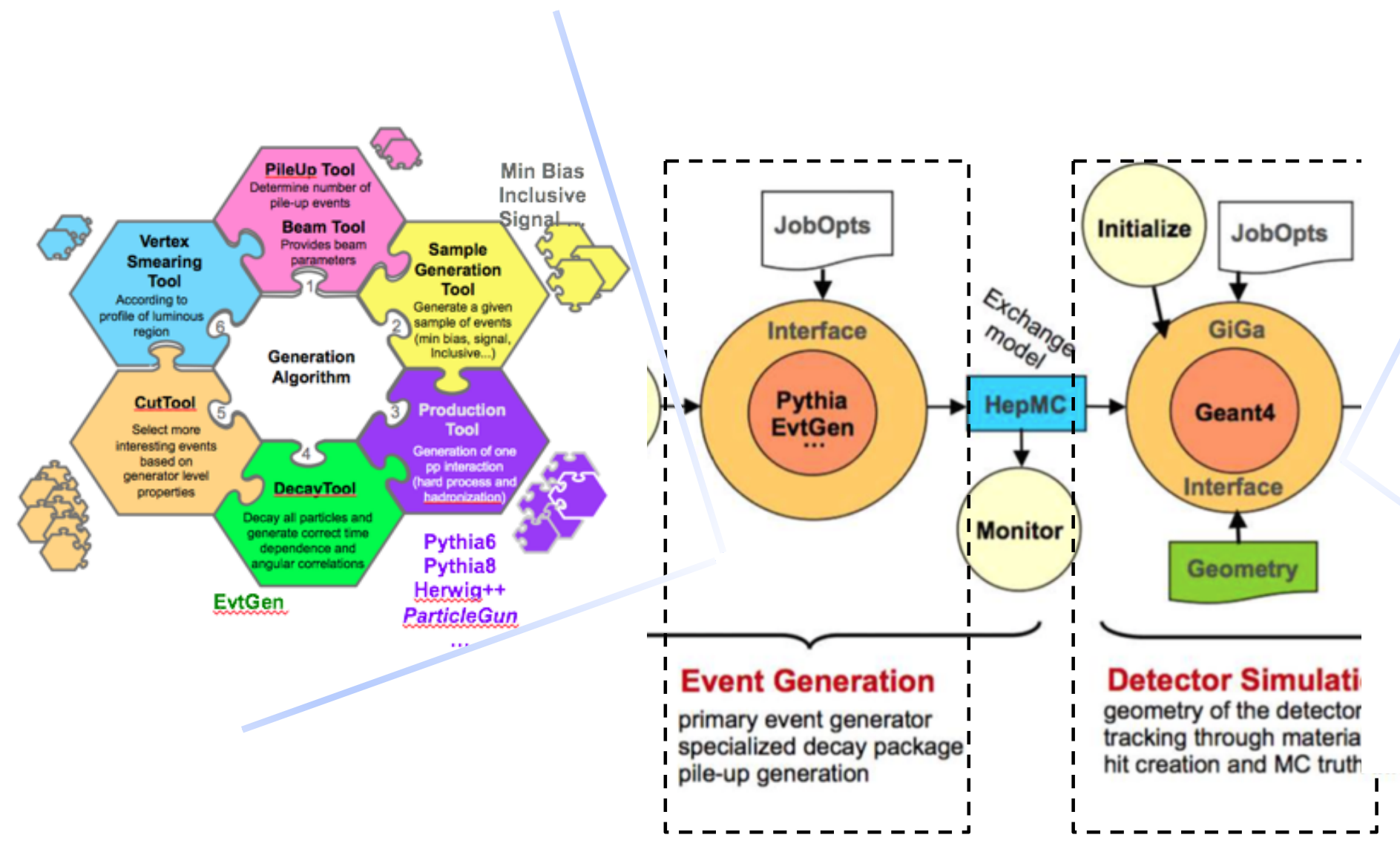

Generator phase with Pythia8 interface implemented Code migrated to HepMC 3

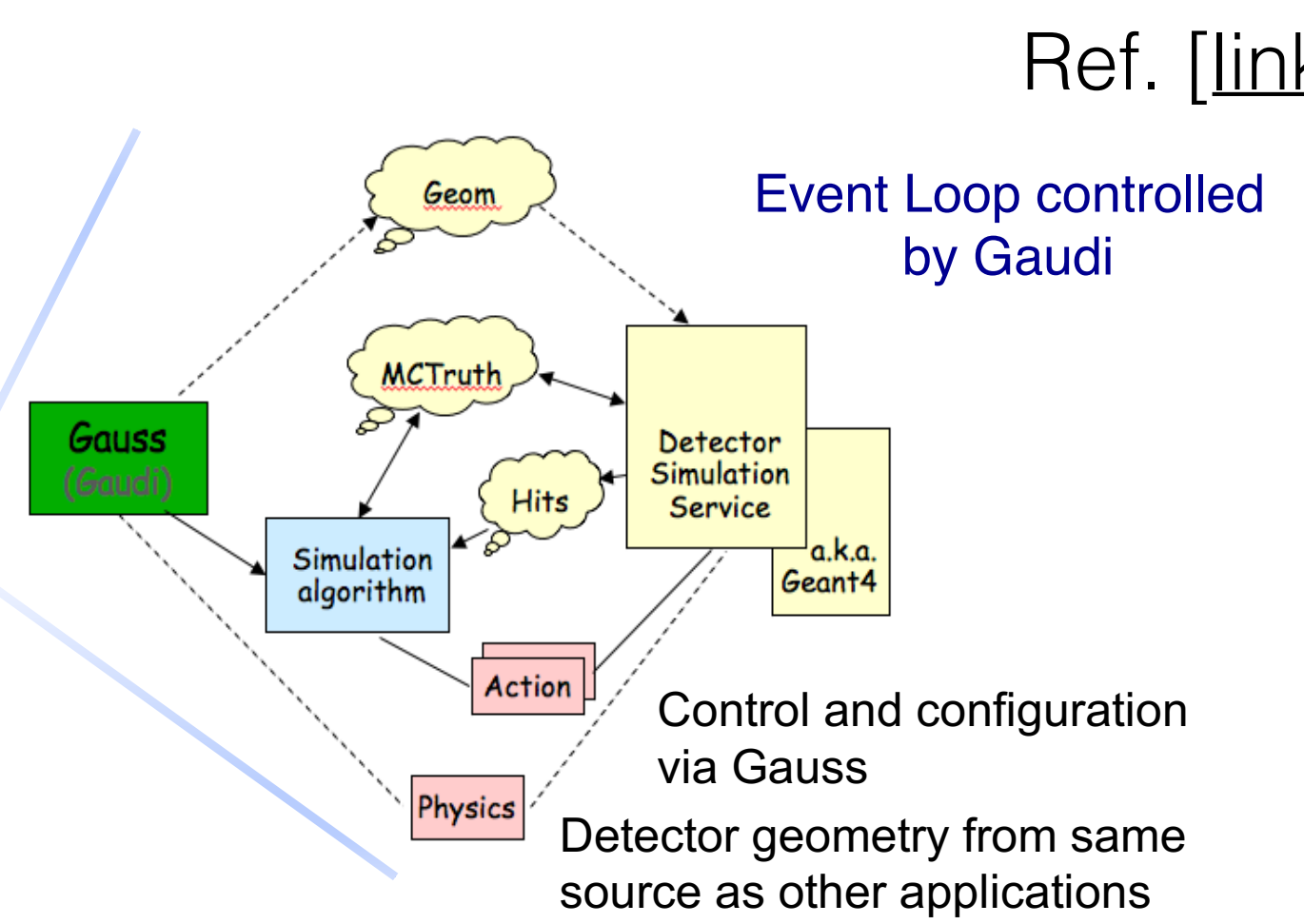

Simulation phase under redesign with identification of elements.

Aim to keep it 'simulation engine' independent 


\section{Execution structure}

Ref. [Task based scheduler]

\section{Gaudi Functional}

- Every Algorithm as task

- Constant execution

- Random Engine created per call

- Fixed input/output, e.g.

/*output data*/ operator()(const /*input data*/) const

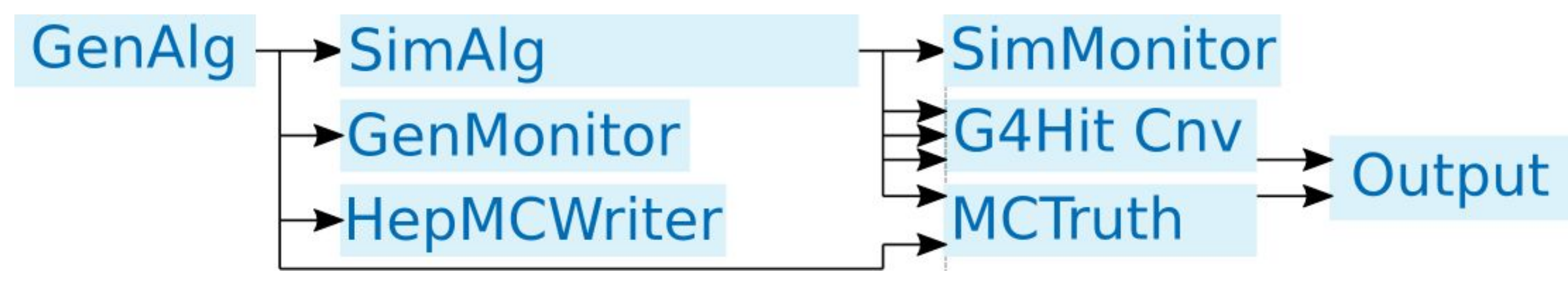




\section{Multi-Threading}

- Thread safety of generators

- Production and decay tools

- Example in Gaussino: Pythia 8

- Shared, locked

- Thread local
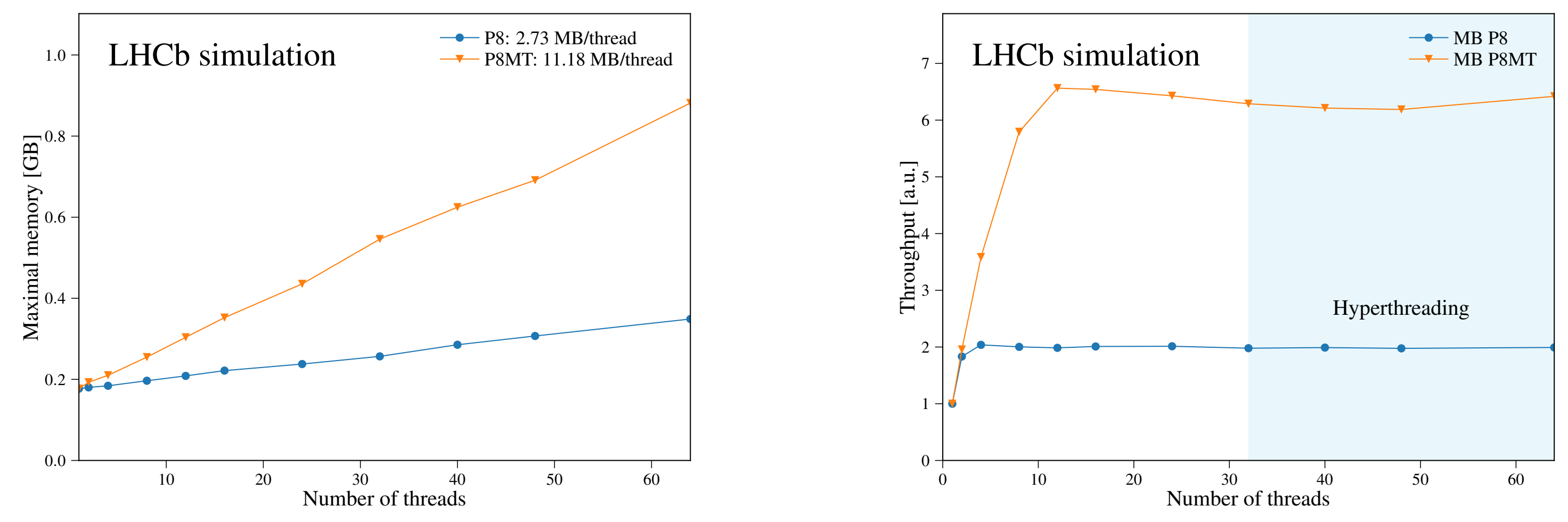

Generation Phase Only!

B. Siddi 


\section{Modular Simulation}

- Take inspiration from the generation: modular!

- A simulation service managing different

backends

- Flexible configuration, e.g. different setting for:

- Pile-up - Spillover - Main Event

- Signal - other particles

- Fast Simulation

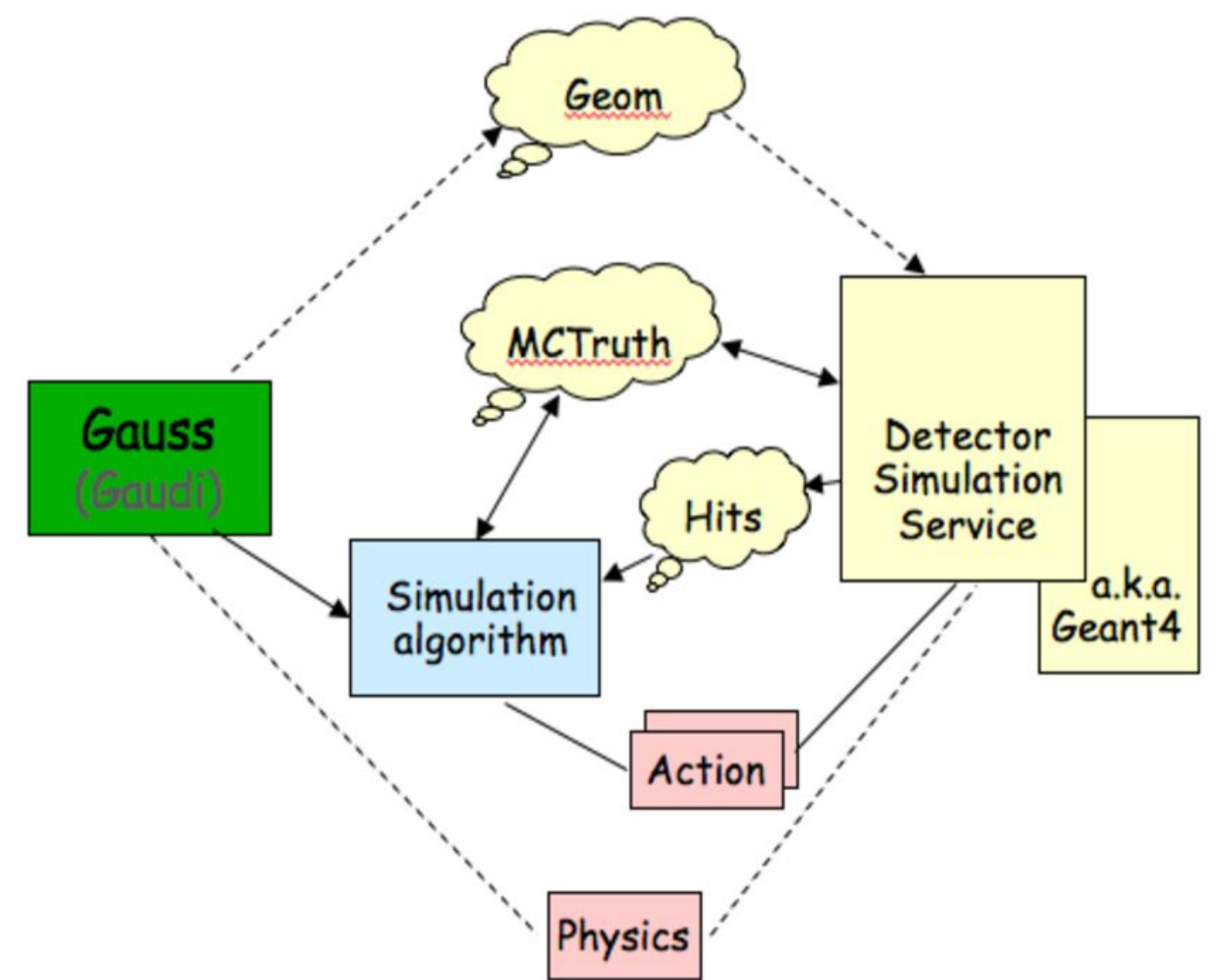




\section{Simulation Interface}

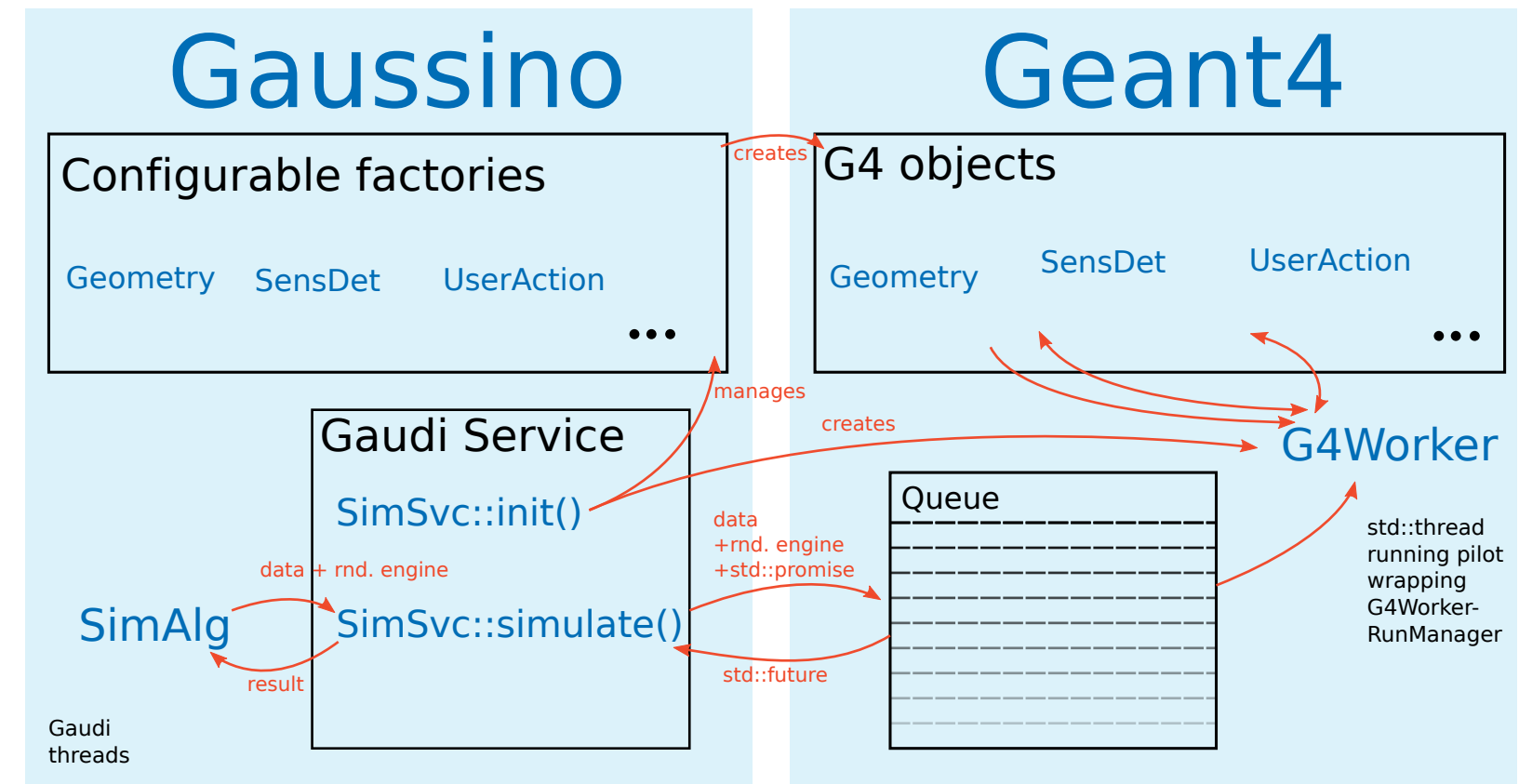

- Gaudi-Tools $\longrightarrow$ factories

- Easy python configuration of G4 settings

- Dynamic assignment possible

- Entire Gaudi event

- Split Gaudi Event into multiple G4 workloads

- Plan to investigate new track based parallelism (in G4) 


\section{Truth Matching}

- Geant4 itself does not have any truth information

\section{In current Gauss}

- Writing HepMC structure keeping the history using G4 track actions

- Relying on global state for communications

- After simulation: converted to MCParticles (object storing the truth in LHCb Event Model)

\section{For Future}

- Redesign for multi-threading

- Simpler: directly write MCParticles 
- Move to DD4Hep for the detector wide translation

- Requires a bit redesign in simulation

\section{User must provide:}

- A Gaudi tool that provides the world pointer

- Logical volume to sensitive detector mapping (configurable via python)

- Basically python configurable UserDetectorConstruction

- DD4hep prototype being tested

\section{Benefits}

- Converter for loaded DD4Hep geometry to Geant4 already exists

- Simplifies the GaussGeo conversion service

- Perform all modifications of geometry in DD4Hep 


\section{Future Gauss prototype}

- Ported additional components

- EvtGen

- LHCb geometry description (DD4Hep in progress)

Added to automatic LHCbPR testing (Ref. [Link]) 


\section{Gauss + Gaussino}

\section{B. Siddi}

INFN Ferrara
Gaussino - a Gaudi-based core simulation framework
LHCb
12 IHCP 


\section{Migrated generation tools}

- EvtGen (single, locking instance)

- Handles all decays

- Logic for signal generation

- LHCb specific generator cuts

- Not affecting the performances
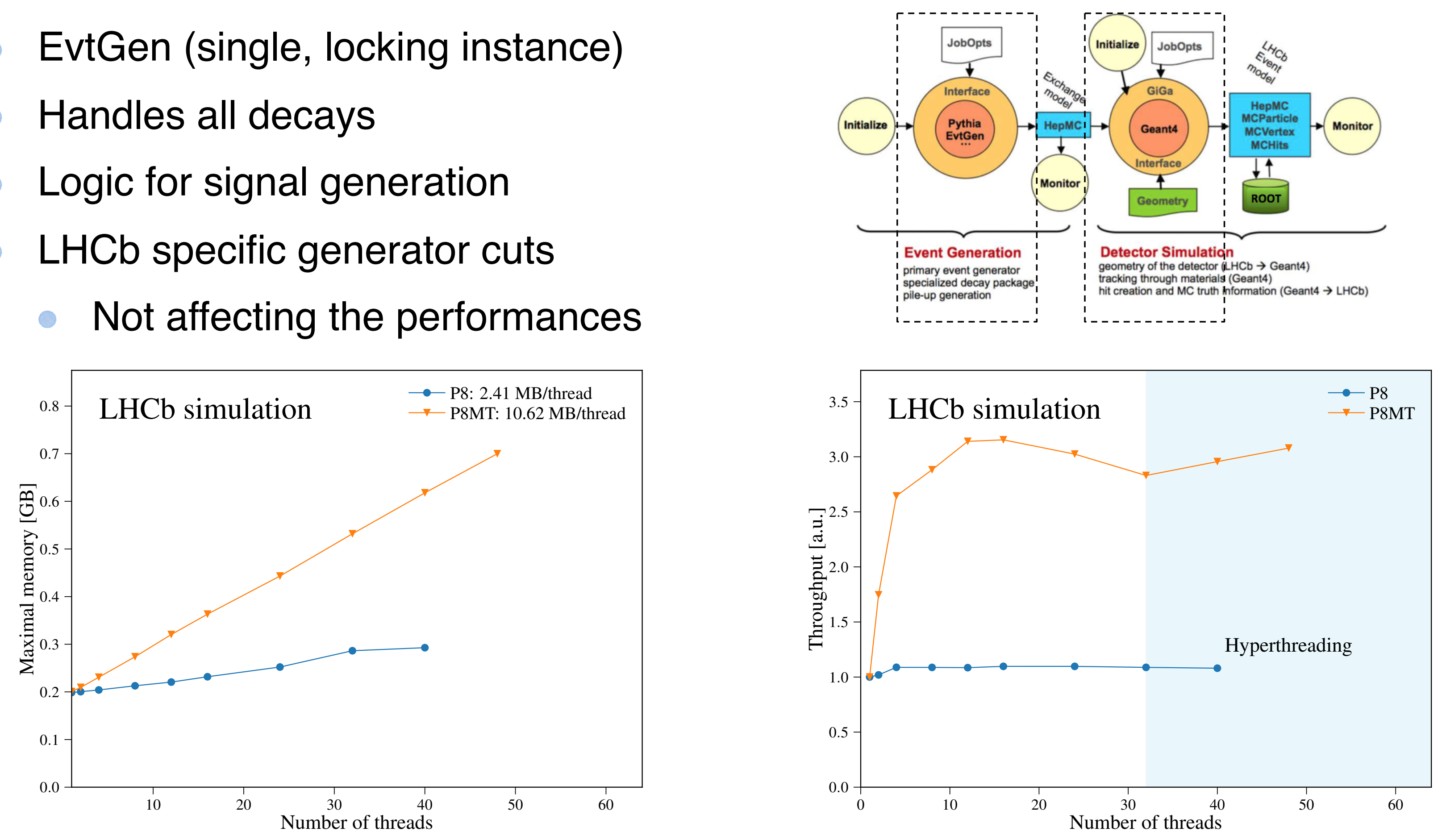

Generation Only $D^{*+} \rightarrow D^{0} \pi^{+}$with $D^{0} \rightarrow K^{-} \pi^{+}$events 


\section{Gauss + Gaussino + Geant4}

\section{B. Siddi}

INFN Ferrara
Gaussino - a Gaudi-based core simulation framework 


\section{Validation studies}

- Performed in LHCbPR (the LHCb performance and regression monitoring tool)
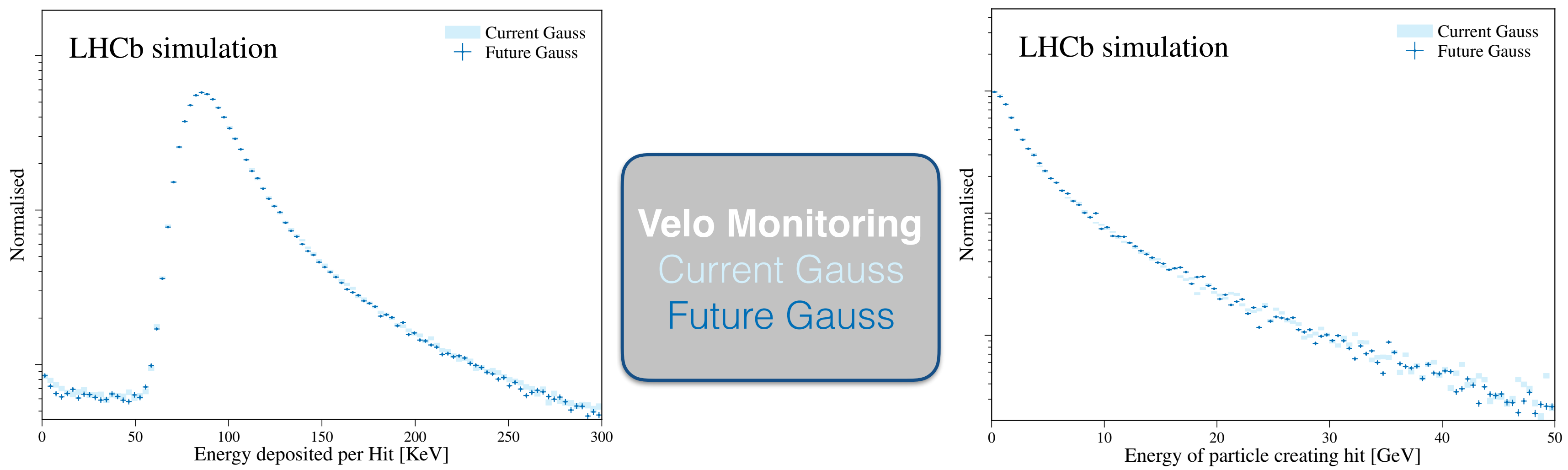

Energy deposit (Generation + Simulation) $D^{*+} \rightarrow D^{0} \pi^{+}$with $D^{0} \rightarrow K^{-} \pi^{+}$events

B. Siddi 


\section{Performances}

- Tested with locking/thread-local generation phase

- Overall good scaling in throughput

- Very good scaling in memory usage

\section{Tested with typical tagged D0 decays with 2016 conditions}
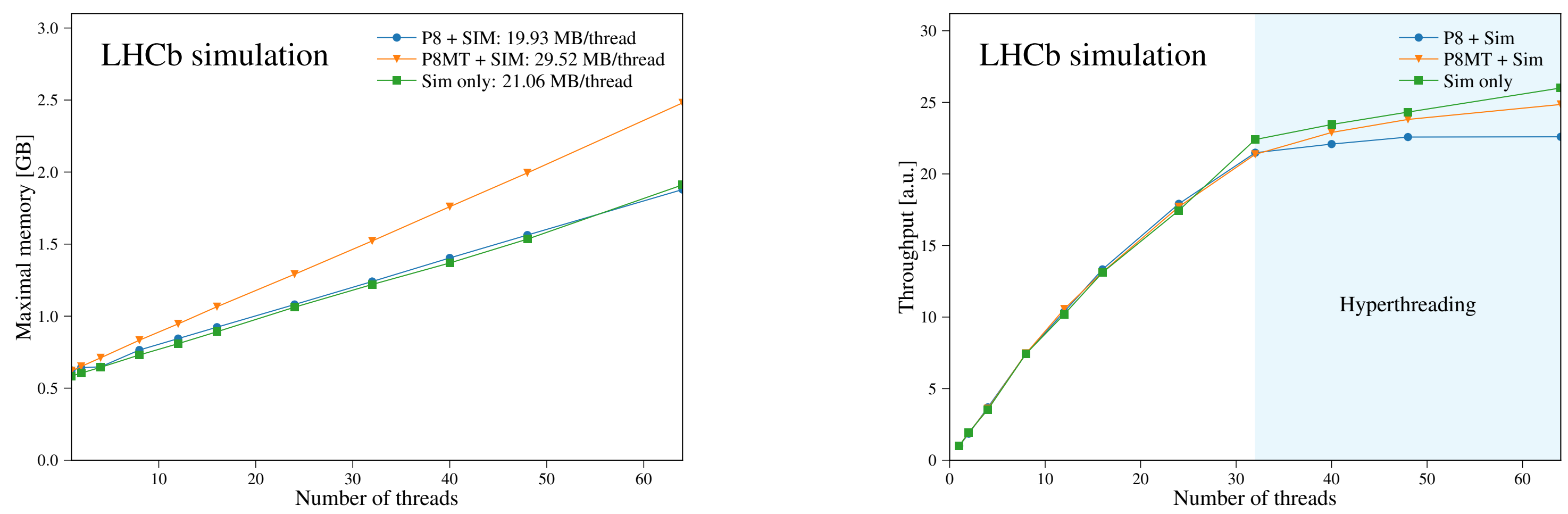

Generation + Simulation $D^{*+} \rightarrow D^{0} \pi^{+}$with $D^{0} \rightarrow K^{-} \pi^{+}$events

B. Siddi 


\section{Conclusions}

- Gaussino, an experiment-independent core framework developed in $\mathrm{LHCb}$ in very advanced state.

- Modular generation phase with Pythia8 example

- Gauss prototype built on top

- Interaction with Geant4 MT

- Encouraging first results, i.e.:

- Performances not affected by LHCb specific tools

- Almost perfect matching between old and new simulation

- Higher throughput 